\title{
Impedance studies and modeling of direct methanol fuel cell anode with interface and porous structure perspectives
}

\author{
Ning-Yih Hsu ${ }^{a}$, Shi-Chern Yen ${ }^{a, *}$, King-Tsai Jeng ${ }^{b}$, Chun-Ching Chien ${ }^{b}$ \\ a Department of Chemical Engineering, National Taiwan University, Taipei 10674, Taiwan \\ ${ }^{\mathrm{b}}$ Institute of Nuclear Energy Research (INER), P.O. Box 3-19, 1000 Wenhua Road, Longtan, Taoyuan 32546, Taiwan
}

Received 17 January 2006; received in revised form 10 March 2006; accepted 28 March 2006

Available online 15 May 2006

\begin{abstract}
In this work, electrochemical impedance was used to analyze the reaction kinetics and interfacial characteristics of an anode in a direct methanol fuel cell (DMFC). An advanced equivalent-circuit model is proposed. The new model incorporates constant phase elements (CPEs) rather than conventional capacitors in the equivalent-circuits taking into account the porous structure of the anode, particularly that in the catalyst layer and at the anode/membrane interface. It effectively simulated the electrochemical characteristics of a DMFC porous anode. The impedance model incorporates the interface factor, resulting in excellent matches between the simulation results and the experimental data in the Nyquist and the Bode plots over a wide range of frequencies. In addition, the differences among methanol electrooxidation reaction kinetics at various operating potentials are clearly observed and satisfactorily explained using electrochemical impedance spectroscopy and the CPE-based equivalent-circuit model.
\end{abstract}

(C) 2006 Published by Elsevier B.V.

Keywords: Direct methanol fuel cell; Dynamic hydrogen electrode; Methanol electrooxidation; Electrochemical impedance spectroscopy; Constant phase element

\section{Introduction}

Direct methanol fuel cells (DMFCs) have attracted increasing attentions as potential portable power sources because of their easy operation, miniaturization and simple fuel supply. The heart of a DMFC is the membrane electrode assembly (MEA). This part may be a Nafion ${ }^{\circledR}$ membrane with catalyzed electrodes bonded onto both sides, resulting in a thin and compact cell structure that favors the fabrication of low-power devices. Although DMFCs face many technical challenges, including catalyst poisoning, methanol crossover and slow reaction kinetics, significant improvements in power densities and cell performances must be achieved for practical applications. These will depend on improvements in the electrode structure and MEA fabrication, as well as advances in electrocatalysts. Therefore, more studies must be conducted on DMFC reaction kinetics and interfacial properties. In particular, such studies that focus on the anode are of critical importance to the development of DMFCs.

\footnotetext{
* Corresponding author. Tel.: +8862 33663052; fax: +886223630397.

E-mail address: scyen@ntu.edu.tw (S.-C. Yen).
}

In studies of reaction kinetics of a DMFC, electrochemical impedance spectroscopy (EIS) is commonly employed [1-4]. Mueller and Urban [5] characterized DMFC processes using ac impedance spectroscopy and identified three arcs on a typical Nyquist plot. At high frequency (from $65 \mathrm{kHz}$ to $900 \mathrm{~Hz}$ ), the size of the arc depended on the temperature and the observed resistance fell as the temperature increased. The intercept of the high-frequency arc on the real-axis was determined by the ionic resistance of the membrane. In the middle of the frequency range $(900-1 \mathrm{~Hz})$, the corresponding resistance depended on the potential and fell as the overpotential increased. This range explained the range of methanol electrooxidation kinetics. The arc was not a semicircle but was quite straight at the higher frequencies, which was caused by the coupling of interfacial and diffusional impedances and the roughness of highly dispersed electrode surfaces. At frequencies of below $1 \mathrm{~Hz}$, the magnitude of the corresponding resistance increased as the methanol flow rate or the concentration fell. Hence, the low-frequency arc was thought to relate to the mass transfer of methanol in the anode. Notably, the third arc in the impedance spectra was observed only when the systems were operated with limited mass transport from an extremely low frequency to $3 \mathrm{mHz}$. 
The Faradaic impedances of DMFC anodes were simulated under kinetically controlled conditions [6,7] by supplying methanol solutions at high flow rates. The capacitorbased equivalent-circuit model employed herein did not include the contributions from the interface and the membrane in ac impedance simulations. The Nyquist plot generally exhibited a response with a single arc or loop, corresponding to electrode kinetics, because of the elimination of mass transfer resistance. However, little information on porous and non-uniform characteristics of the catalyst layer is available and previous simulation models did not take into account the effects of the membrane and the electrode interface on the impedance simulations. The results of these simulations have large discrepancies. This investigation studies both sectors of the membrane and the electrode interface over a wide range of frequencies in the impedance simulations.

In ac impedance studies, most equivalent-circuit models are based on the Randles circuits using RC elements with some modifications. A double-layer capacitor is generally utilized to model the interfacial phenomena of the electrodes. However, the use of capacitive elements in equivalent-circuits usually causes discrepancies in fitting the experimental data; in particular, with respect to the complex methanol electrooxidation process in a DMFC, mainly due to the use of capacitive elements that over-simplifies the treatment of the interfacial properties in the catalyst layer. The complete anode reaction of a DMFC consists of three parts - the catalyst layer at the anode, the anode/membrane interface and the proton exchange membrane - so a more complete model must account for all of these parts. In fact, the membrane/electrode interface and the catalyst layer have unique structures that are quite porous and rough. Accordingly, they cannot be perfectly simulated as ideal capacitors. Rather, frequency-dependent elements, such as constant phase elements (CPEs), are normally required to simulate such nonideal conditions.

$\mathrm{CPE}$ is a hypothetical element that was introduced by Cole and Cole [8] to make the equivalent-circuit model more consistent with the actual processes that occur across the electrochemical interfaces. It is equivalent to a combination of many $\mathrm{RC}$ sections with various time constants. Therefore, it has the following advantages: (a) it simplifies a complicated equivalentcircuit model and does not depend on the use of an excessive number of electrical elements, and (b) it increases the accuracy and usefulness of the illustration of the investigated properties because it uses a simpler equivalent-circuit. The impedance of a CPE is expressed as [9]:

$Z=1 /\left[Q(j \omega)^{p}\right]$

where $Q$ is the admittance constant and $p$ is an adjustment parameter. The value of $p$ for an electrode or an interface is typically between 0.5 and 1.0. In the limiting case of $p=1.0$, the equation represents a capacitor. At the other extreme case of $p=0$, it is identical to that of a resistor. Hence, a smaller $p$ corresponds to a greater deviation of the element from a perfect capacitor. Table 1 summarizes the characteristics of CPEs at various $p$ values. A $\mathrm{CPE}$ is commonly adopted in a model in place of a capacitor to
Table 1

Characteristics of constant phase element [10]

\begin{tabular}{ll}
\hline Generalized expression & $Z=1 /\left[Q(j \omega)^{p}\right]$ \\
\hline Capacitor & $p=1 ; Z=1 /[Q(j \omega)]$ \\
Resistor & $p=0 ; Z=1 / Q$ \\
Warburg element & $p=0.5 ; Z=1 /\left[Q(j \omega)^{0.5}\right]$ \\
A porous electrode or rough interface & $0.5<p<1.0 ; Z=1 /\left[Q(j \omega)^{p}\right]$ \\
\hline
\end{tabular}

compensate for the heterogeneity of an electrode or an interface in the system. In fact, a capacitor is a special case of a constant phase element-one with a constant phase angle of $90^{\circ}$ degrees. A CPE with $p=0.5$, however, can be adopted to generate an infinitely long Warburg element, which applies when the charge carrier diffuses into a porous material but does not fully penetrate the layer under low-frequency response conditions.

The CPE has become a powerful tool in equivalent-circuit simulations. Berthier et al. [10] demonstrated the theoretical treatment of equivalent-circuits that include CPEs. Recently, CPE has been widely used in simulations of a range of electrochemical systems of practical importance, such as batteries $[11,12]$, metal corrosion $[13,14]$, electrode interfaces and thin films [15-19], electrode reactions [20,21], electrochemical sensors [22], PEM fuel cells [23] and others. Given such important applications, however, so far no studies that included CPEs in simulations of DMFC systems can be found. Accordingly, this study proposes a new CPE-based electrochemical impedance approach to eliminate the apparent shortcomings of the use of capacitive elements in simulations of DMFC anodes reported previously [5-7]. Briefly, the CPEs adjusted the perfect capacitive elements that were frequently employed to describe the interfacial double layer and the catalyst layer under more realistic conditions, to something between perfect resistors and capacitors, according to the porosity of the catalyst layer or the roughness of the interfaces. Fig. 1 illustrates the porous structure of a DMFC anode in conjunction with a porous or rough interface. These two porous-structure regions are treated separately due to different physical and electrochemical properties. In the catalyst layer, the deep layer is more compact and more difficult

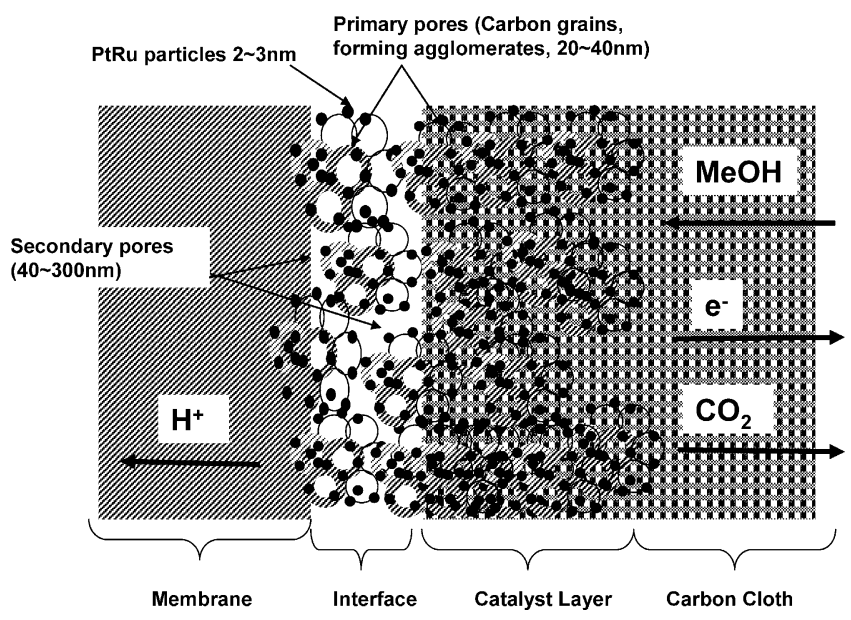

Fig. 1. shows the porous structure of a DMFC anode in conjunction with a porous interface. 


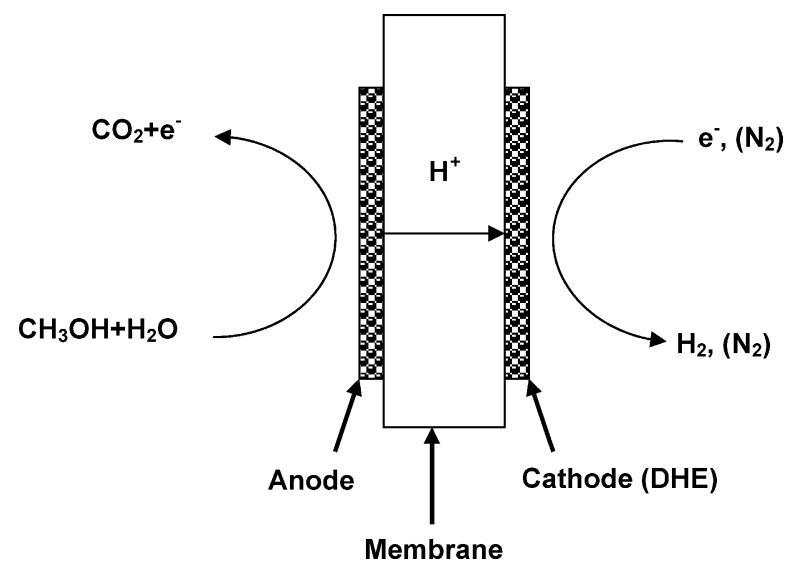

Fig. 2. The schematic diagram of a DMFC coupled with a dynamic hydrogen electrode (DHE) for electrochemical impedance measurements.

to access than the outer layer. A similar electrode structure can be found in a previous report [24].

In investigations of hydrogen-air fuel cells, oxygen cathode impedances constitute most of the measured cell impedances [25], because of the fast kinetics and ease of mass transport in the hydrogen oxidation reaction, such that the impedance contribution of the hydrogen anode is normally negligible. A hydrogen evolution reaction (HER), presented in Fig. 2, is employed as the counter electrode in this study to eliminate the effect of impedance to the cathode at which oxygen is reduced. In the ac impedance experiments, the hydrogen evolution reaction at the cathode can also be applied to establish a dynamic hydrogen electrode (DHE) reference. This setup provides a favorable experiment environment and considerably simplifies the measurement procedures. Notably, based on this configuration, the cell is basically a pseudo-DMFC with the anode electrooxidizes methanol and the cathode functions as a DHE.

\section{Impedance models}

\subsection{Conventional equivalent-circuit model of the Faradaic impedance of a DMFC anode}

In the work of the Faradaic impedance of a DMFC anode using a conventional equivalent-circuit model, the most commonly adopted mechanism for the electrooxidation of methanol can be described as follows [6,7]:

$\mathrm{CH}_{3} \mathrm{OH} \stackrel{[\mathrm{Pt}] r_{1}}{\longrightarrow}(\mathrm{CO})_{\mathrm{ads}}+4 \mathrm{H}^{+}+4 \mathrm{e}^{-+\mathrm{H}_{2} \mathrm{O}[\mathrm{Ru}] r_{2}} \mathrm{CO}_{2}+2 \mathrm{H}^{+}+2 \mathrm{e}^{-}$

Methanol is adsorbed and reacted, mainly on Pt active sites, with a rate $r_{1}$ to yield $(\mathrm{CO})_{\text {ads }}$ as the dominating intermediate species. The oxidative removal of adsorbate at a rate $r_{2}$, in conjunction with the dissociation of $\mathrm{H}_{2} \mathrm{O}$ at $\mathrm{Ru}$ active sites, is the rate-determining step under conditions that apply during the operation of a methanol fuel cell; these conditions include low anode potentials $(E<0.4 \mathrm{~V}$ [RHE]) and moderately high temperatures. The net rate of production of electrons, re, and the net rate of production of $(\mathrm{CO})_{\mathrm{ads}}, r_{\mathrm{CO}}$, could be defined as follows $[6,7]$ :

$r_{\mathrm{e}}=4 r_{1}+2 r_{2}=\frac{i}{F}$

$r_{\mathrm{CO}}=r_{2}-r_{1}=\frac{q_{\mathrm{CO}}}{F} \frac{\mathrm{d} \theta}{\mathrm{d} t}$

where $q_{\mathrm{CO}}$ is the charge required for adsorption of $\mathrm{CO}$ to complete coverage, $\theta$ the fractional surface coverage of $\mathrm{CO}$ and $F$ is the Faraday constant. This conventional Faradaic impedance model deals only with electrode reaction kinetics and does not take into account the effects of the membrane and the interface.

\subsection{Conventional equivalent-circuit model of a DMFC anode with membrane only (model A)}

The DHE is located at the opposite side of the membrane from the anode, so the measured impedances include those associated with the membrane. Fig. 3(a) shows the equivalent-circuits, where $R_{\mathrm{m}}$ is the resistance of the membrane, $R_{\mathrm{ct}}$ the charge transfer resistance, $C_{\mathrm{d}}$ the double-layer capacitance, and $R_{\mathrm{c}}$ represents the resistance of the solid phase in catalyst layer. The inductance $L_{\mathrm{CO}}$ is such that the current signal follows a voltage perturbation with a phase delay because of the slowness of the relaxation of $(\mathrm{CO})_{\text {ads }}$ coverage. The simulation modeling with respect to the kinetics-controlled region had been proposed in several reports [5-7].

\subsection{Conventional equivalent-circuit model of a DMFC anode in conjunction with both membrane and interface (model B)}

The DHE is located at the opposite side of the membrane from the anode, so the measured impedances include those associated with the membrane and the interface at the anode side. Fig. 3(b) depicts the more completed equivalent-circuits, taking into account these two contributions. In the figure, $R_{\mathrm{m}}$ denotes the resistance of the membrane and $R_{\mathrm{i}}$ represents the resistance of the interface between the membrane and the catalyst layer.

\subsection{CPE-based equivalent-circuit model of a DMFC anode in conjunction with both membrane and interface (model C)}

Notably, the electrodes of interest often exhibit flattening of the measured spectrum at a specific potential, probably caused by the roughness or the porosity of the structure of the catalytic layer. The interface considered in this case may be governed by the roughness and the imperfect contact between membrane and the catalyst layer. If the effects of the roughness of the catalytic layer and the non-uniform distribution of the catalyst are considered, then the impedance of the $\mathrm{Pt}-\mathrm{Ru} / \mathrm{C}$ anode typically exhibits a non-semicircular response in the Nyquist plots. Therefore, the conventional equivalent circuits in Fig. 3(b) do not yield results that fit the experimental results. The developed new model incorporates constant-phase elements rather than ideal capacitors, as shown in Fig. 3(c), but applies the same reaction 


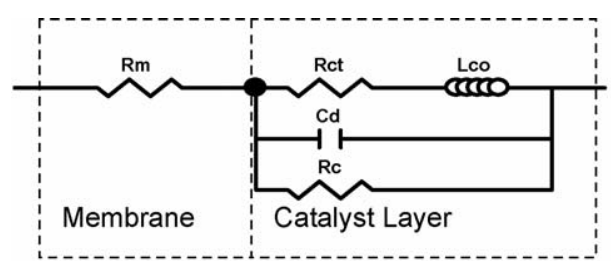

(a) Conventional model (Model A)

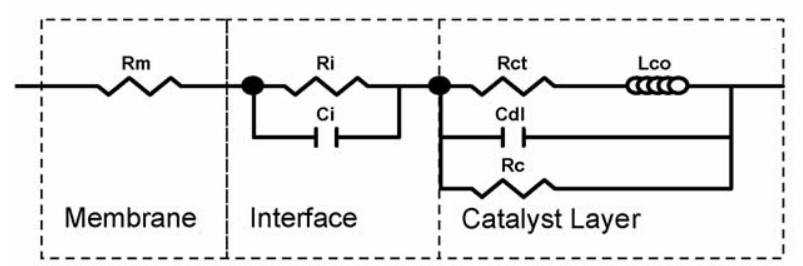

(b) Proposed capacitor-based model with interface (Model B)

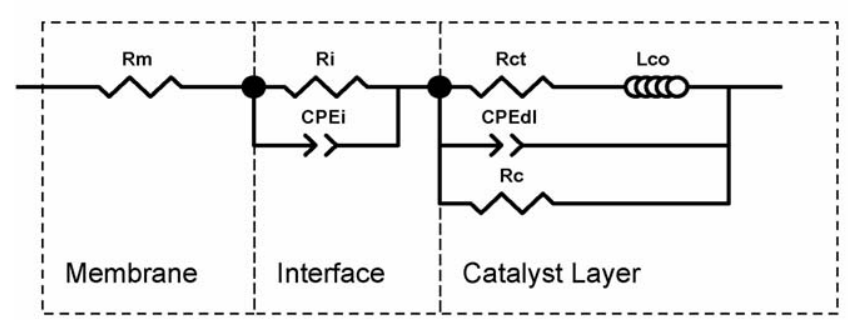

(c) Proposed CPE-based model with interface (Model C)

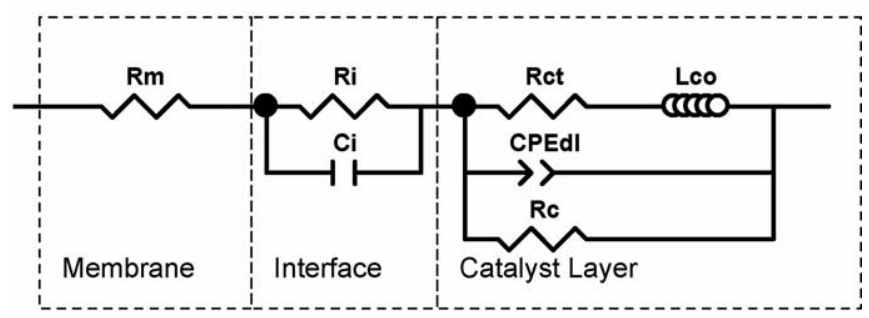

(d) Model $\mathrm{C}$ without a CPE for the membrane interface (Model D)

Fig. 3. The equivalent-circuits for modeling the impedances of a DMFC anode: (a) conventional equivalent-circuit of membrane and catalyst layer only (model A); (b) Ideal capacitor incorporated equivalent-circuit of membrane + interface + catalyst layer (model B); (c) CPE incorporated equivalentcircuit of membrane + interface + catalyst layer (model C); (d) model C without a CPE at the interface (model D).

mechanism. The model incorporates two CPEs to account for the anode-membrane interface and the catalyst layer, respectively. Hence, the complex impedance of the equivalent-circuits of the interface $\left(Z_{\text {interface}}\right)$ can be expressed as

$Z_{\text {interface }}=\frac{R_{\mathrm{i}}}{1+R_{\mathrm{i}} Q_{\mathrm{i}}(j \omega)^{p_{\mathrm{i}}}}$

where $p_{\mathrm{i}}$ is the adjustment parameter of the CPE and $Q_{\mathrm{i}}$ is the admittance constant at the interface. Similarly, the complex impedance of the equivalent-circuits of the catalyst layer $\left(Z_{\text {catalyst layer }}\right)$ can be expressed as

$Z_{\text {catalyst layer }}=\frac{R_{\mathrm{c}}\left(R_{\mathrm{ct}}^{2}+\omega^{2} L_{\mathrm{CO}}^{2}\right)}{1+R_{\mathrm{c}}\left[R_{\mathrm{ct}}-j \omega L_{\mathrm{CO}}+Q_{\mathrm{dl}}(j \omega)^{p_{\mathrm{dl}}}\right]}$ where $p_{\mathrm{dl}}$ is the adjustment parameter of the CPE, $L_{\mathrm{CO}}$ the inductance element and $Q_{\mathrm{dl}}$ is the admittance constant in the catalyst layer. The overall impedance is simply the summation of the specific impedance in each section.

\subsubsection{Model $C$ without a CPE at the interface (model D)}

In order to eliminate the possible interfaces of using two CPEs simultaneously in a model, the CPE at the interface of model C was replaced with an ideal capacitor forming model $\mathrm{D}$ as shown in Fig. 3(d).

\section{Experimental}

The experimental setup as shown earlier in Fig. 2 is used to measure the methanol electrooxidation of a DMFC anode at various potentials. The electrodes were prepared as follows: (a) a carbon paper (Toray Co.) was treated to make it hydrophobic; (b) a thin PTFE/carbon layer was cast onto the treated carbon paper surface; (c) the catalyst layer was cast onto the surface of the supporting layer. The catalyst suspension comprised $\mathrm{Pt} / \mathrm{C}$ or $\mathrm{Pt}-\mathrm{Ru} / \mathrm{C}$ (Johnson Matthey) catalyst powder, 5 wt.\% Nafion ${ }^{\circledR}$ solution (DuPont) and ethylene glycol (EG). The finely ground catalyst powder was first mixed with EG, and then Nafion ${ }^{\circledR}$ solution was slowly added with stirring. Then, the mixture was agitated by ultrasonication using a sonicator (XL2020, Heat Systems Inc.). Finally, the catalyst suspension was applied to a carbon paper support by casting to yield a catalyst layer with a thickness of $50 \mu \mathrm{m}$. The catalyst loading of the prepared anode was $3 \mathrm{mg} \mathrm{Pt}-\mathrm{Ru} \mathrm{cm}{ }^{-2}$ and that of the cathode was $1.5 \mathrm{mg} \mathrm{Pt} \mathrm{cm}^{-2}$. In the preparation of a membrane electrode assembly (MEA), the electrodes were hot-pressed onto both sides of a Nafion ${ }^{\circledR} 117$ membrane at $130{ }^{\circ} \mathrm{C}$ under an applied pressure of $0.5 \mathrm{kN} \mathrm{cm}^{-2}$ for $90 \mathrm{~s}$. The prepared MEA was placed in an oven at $130^{\circ} \mathrm{C}$, which was evacuated for $12 \mathrm{~h}$ to remove the incorporated EG. Then, the treated MEA was fabricated into a single-cell DMFC using two graphite endplates and fastened with bolts and nuts. In the experiments, pure oxygen was first used in the cathode compartment as an oxidant to form a true single-cell DMFC. After the DMFC had reached a stable condition, the oxygen was replaced with nitrogen, to form a DMFC coupled with a DHE. The single-cell DMFC was fed with $2 \mathrm{M}$ methanol solution by circulation on the anode side at $40{ }^{\circ} \mathrm{C}$ and atmospheric pressure. Electrochemical experiments were undertaken over a range of potentials from 0.3 to $0.5 \mathrm{~V}$ (DHE) with good mass-transport, so the effect of mass-transport on impedance was negligible.

The frequency generator/analyzer was a Solartron SI 1260 Impedance Spectroscope controlled using a personal computer and coupled to a potentiostat (Solartron 1286), to support the modulation of large dc currents. The amplitude of the sinusoidal potential signal was $10 \mathrm{mV}$. Experimental data were collected between $10 \mathrm{kHz}$ and $5 \mathrm{mHz}$ frequencies and electrochemical impedance spectra were obtained accordingly. The value of the parameter of each electrical circuit element was determined using Z-View software (Scribner Associates) from the experimental data. The simulation modeling procedure and method are detailed in the operation manual of Z-View Software. Briefly, 
Table 2

Fitting parameters for the new equivalent-circuit on modeling the DMFC anode using constant phase elements at various potentials

\begin{tabular}{|c|c|c|c|c|c|c|c|c|c|}
\hline Potential (V/DHE) & $R_{\mathrm{m}}\left(\Omega \mathrm{cm}^{2}\right)$ & $\mathrm{CPE}_{\mathrm{i}}, Q\left(\mathrm{~F} \mathrm{~cm}^{-2}\right)$ & $\mathrm{CPE}_{\mathrm{i}}, p$ & $R_{\mathrm{i}}\left(\Omega \mathrm{cm}^{2}\right)$ & $R_{\mathrm{ct}}\left(\Omega \mathrm{cm}^{2}\right)$ & $L_{\mathrm{CO}}\left(\mathrm{H} \mathrm{cm}^{-2}\right)$ & $\mathrm{CPE}_{\mathrm{dl}}, Q\left(\mathrm{~F} \mathrm{~cm}^{-2}\right)$ & $\mathrm{CPE}_{\mathrm{dl}}, p$ & $R_{\mathrm{c}}\left(\Omega \mathrm{cm}^{2}\right)$ \\
\hline 0.3 & 0.701 & 0.076 & 0.572 & 3.368 & 2.903 & 3.934 & 0.949 & 0.992 & 5.531 \\
\hline 0.4 & 0.692 & 0.071 & 0.586 & 2.489 & 2.992 & 0.982 & 0.872 & 0.985 & 2.089 \\
\hline 0.5 & 0.682 & 0.071 & 0.598 & 1.762 & 7.600 & 0.516 & 0.518 & 0.757 & 1.818 \\
\hline
\end{tabular}

after collecting the experimental data, the models to be simulated are defined forming various equivalent circuits. Various elements can be employed in the equivalent circuits. Initial estimates for all elements are entered and the element values to be calculated are selected. Simulation is performed based on an instant-fit approach. Simulations were carried out between $5 \mathrm{mHz}$ and $10 \mathrm{kHz}$. It should be noted that all the errors of the modeling parameters are within $1 \%$ in this study. This confirms the accuracy of our modeling.

\section{Results and discussion}

\subsection{Effect of membrane on impedance}

Table 2 shows that the resistance at the point at which the frequency first became high $(10 \mathrm{kHz})$ indicates that of the proton exchange membrane $\left(R_{\mathrm{m}}\right)$, which is around $0.7 \Omega \mathrm{cm}^{2}$ and independent of the operating potential. This value is equivalent to a conductivity of $0.025 \Omega^{-1} \mathrm{~cm}^{-1}$ for the Nafion ${ }^{\circledR} 117 \mathrm{mem}$ brane and is similar to the reported value of $0.01-0.03 \Omega^{-1} \mathrm{~cm}^{-1}$ [26,27].

\subsection{Polarization and impedance of direct methanol anode with membrane}

Fig. 4 plots the curve of polarization of the DMFC anode during the electrooxidation of methanol. The figure reveals that the employed DMFC suffered from a fairly large anodic polarization loss, which is common, and only began to generate a significant current density of over $0.2 \mathrm{~V}$ (DHE). However, no mass-transport losses can be found with the polarization potential up to $0.6 \mathrm{~V}$ (DHE). Fig. 5 plots the measured impedance data

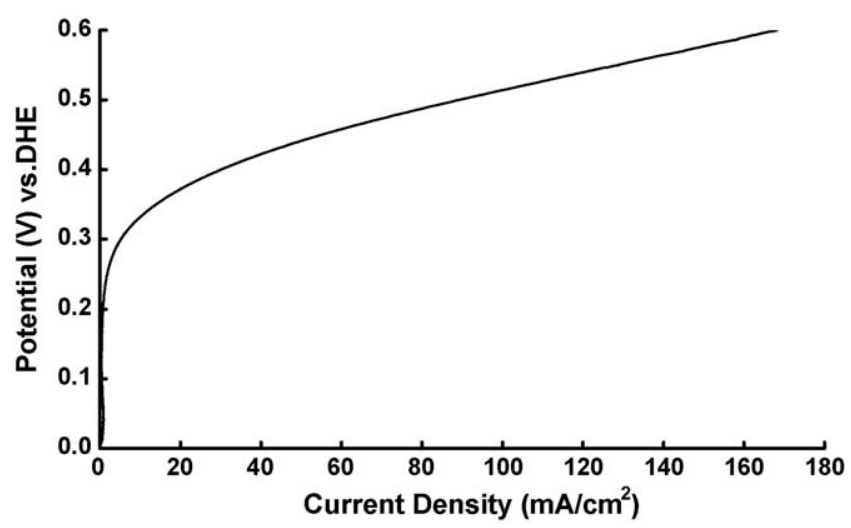

Fig. 4. The polarization curve of the DMFC anode (DHE) obtained at $40^{\circ} \mathrm{C}$, $2 \mathrm{M} \mathrm{MeOH}$ and ambient pressure. Anode catalyst loading was $3 \mathrm{mg} \mathrm{Pt}-\mathrm{Ru} \mathrm{cm}^{-2}$ and the cathode was $1.5 \mathrm{mg} \mathrm{Pt} \mathrm{cm}{ }^{-2}$. The ohmic drop is corrected. at various potentials. When a potential of $0.3 \mathrm{~V}(\mathrm{DHE})$ is applied to the DMFC anode (which potential is low, and one at which the electrooxidation of methanol is slow and reaction intermediates are generated), a large amount of $\mathrm{CO}$ is adsorbed on the surfaces of the Pt-Ru catalyst, resulting in a strong inductance effect. When the operating potential is $0.4 \mathrm{~V}$ (DHE), the loop at the low frequency becomes smaller (cf. Fig. 5), suggesting less $\mathrm{CO}_{\text {ads }}$ is reacted at the surface of the electrode. As the applied potential is increased further to $0.5 \mathrm{~V}$ (DHE), the methanol electrooxidation rate increases and the adsorbed $\mathrm{CO}$ is almost all reacted to $\mathrm{CO}_{2}$ as a final product, according to Eq. (2). Hence, the very small amount of $\mathrm{CO}_{\mathrm{ads}}$ formed on Pt-Ru surfaces causes the almost complete disappearance of inductance, as demonstrated in Fig. 5 and Table 2. This range of potentials is, in fact, the most commonly used range for a DMFC.

\subsection{Nyquist and bode plots of various equivalent-circuit models}

Fig. 6 presents the comparisons of the impedance plots of a Pt-Ru/C anode at $0.3 \mathrm{~V}$ (DHE) for experimental data and fittings of three impedance models. The experimental data reveal one arc and one loop. The arc is attributable to the response of the interface, which is superimposed into the loop. However, the loop is caused mainly by the Faradaic impedance response of the methanol electrooxidation to the inductance caused by $\mathrm{CO}_{\mathrm{ads}}$ on the catalyst surfaces, as revealed elsewhere $[6,7]$. The arc in the high frequency region is not a regular semicircle, but points upward at the intercept of the high frequency end, becoming flattened at below $1 \mathrm{~Hz}$, under the influence of coupling between the interfacial impedance and the impedance of membrane, that is strongly influenced by the roughness or porosity of the anode/membrane interface. Accordingly, the impedance

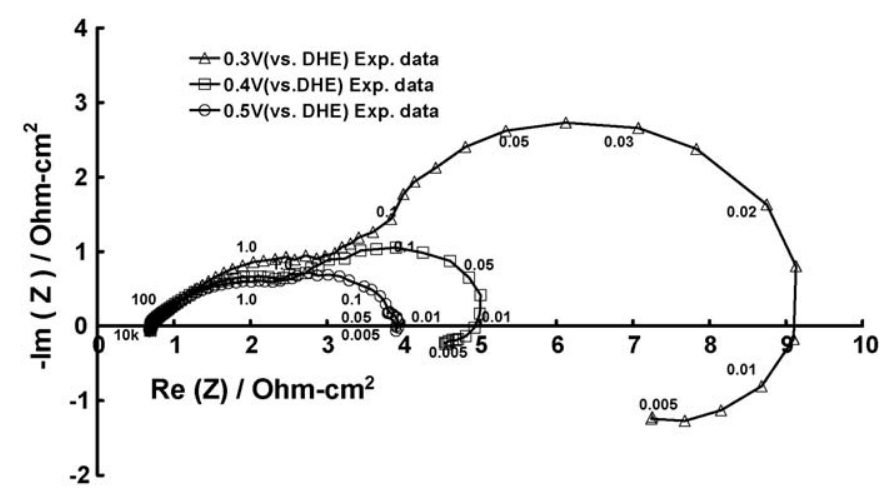

Fig. 5. The Nyquist plots of a Pt-Ru/C anode at various potentials (DHE) obtained at frequencies between $10 \mathrm{kHz}$ and $5 \mathrm{mHz}$, at $40^{\circ} \mathrm{C}, 2 \mathrm{M} \mathrm{MeOH}$ and ambient pressure. Anode: $3 \mathrm{mg} \mathrm{Pt}-\mathrm{Ru} \mathrm{cm}^{-2}$; cathode: $1.5 \mathrm{mg} \mathrm{Pt} \mathrm{cm}^{-2}$. 


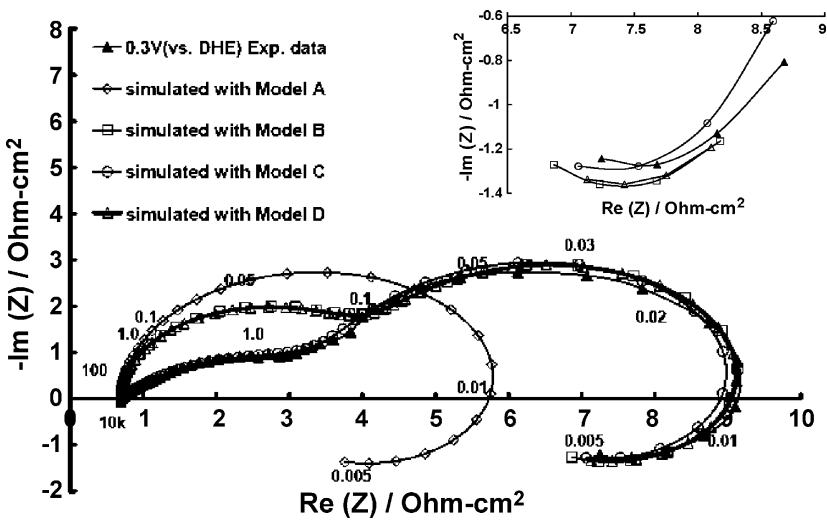

Fig. 6. Comparisons of the impedance plots of a Pt-Ru/C anode at $0.3 \mathrm{~V}(\mathrm{DHE})$ for experimental data and fittings of impedance models. The inset illustrates the detailed fittings at the kinetics-controlled region.

model described in the literature [5-7] is modified herein, and the equivalent-circuit models including models $\mathrm{B}$ and $\mathrm{C}$ proposed herein includes an interface region. At frequencies of under $0.1 \mathrm{~Hz}$, the upward arc of the loop is almost semicircular. However, the magnitude of the corresponding resistance is quite large; indicating that at this moderate potential, the driving force in the methanol charge-transfer reaction is still not sufficiently large to facilitate the further oxidation of $\mathrm{CO}$ on the surfaces of the electrode. Furthermore, the largeness of the loop further shows that the surfaces adsorb substantial amount of $\mathrm{CO}$ and, perhaps, other methanol electrooxidation intermediates. The species adsorbed onto the electrocatalyst surfaces forms an insulating dielectric that causes high resistance. It also inhibits the electrooxidation of methanol and increases resistance. The magnitude of the resistance and the phase angle can be easily analyzed using Bode plots. Fig. 7 presents corresponding Bode plots of resistance magnitude and phase angle against frequency.

The usefulness of the model $\mathrm{C}$ depends on the goodness of fit between the experimental data and the simulated results. Fig. 6 shows that the experimental data almost perfectly match the simulation using CPEs over the whole range of frequencies. However, capacitive elements in the equivalent-circuit model provide a fairly good fit with the kinetics of the electrode, but

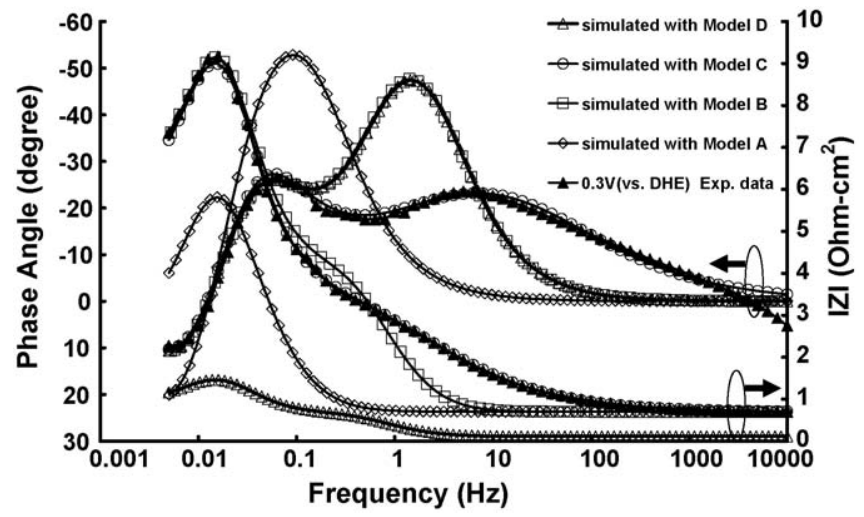

Fig. 7. Comparisons of the Bode plots of a Pt-Ru/C anode at $0.3 \mathrm{~V}$ (DHE) for experimental data and fittings of impedance models.

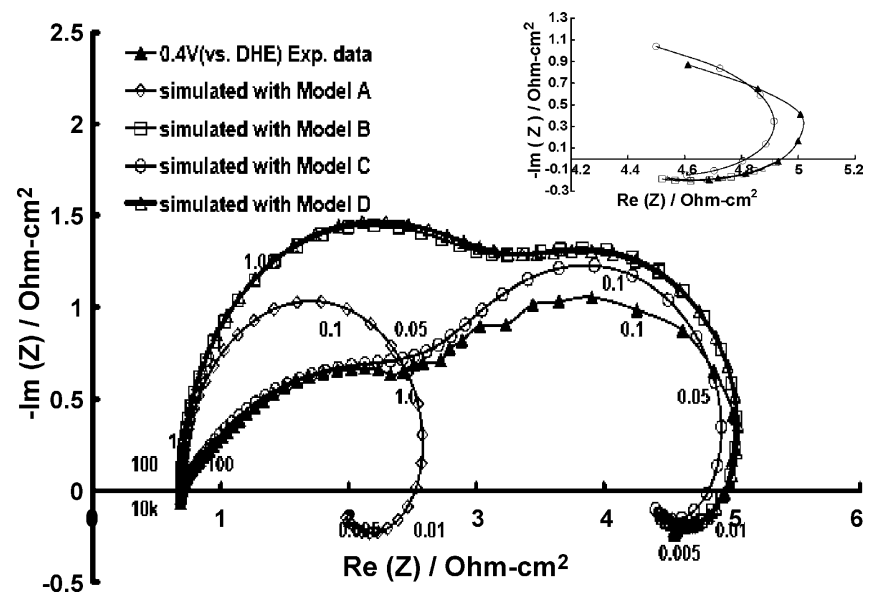

Fig. 8. Comparisons of the Nyquist impedance plots of a Pt-Ru/C anode at $0.4 \mathrm{~V}$ (DHE) for experimental data and fittings of impedance models. The inset illustrates the detailed fittings at the kinetics-controlled region.

not with the interface-related kinetics. Clearly, the characteristics of the interface considered in the CPE-based model C strongly affect this part even at a moderate reaction potential of $0.3 \mathrm{~V}$ (DHE), suggesting the importance of using an interface section between the membrane and the electrode, in the electrochemical impedance simulations. Similarly, the Bode plot shows the goodness of fit of the proposed impedance model C, while the capacitive element-based model B yields large mismatches with the impedance responses of the interface over the frequency range of interest.

As the potential is increased to $0.4 \mathrm{~V}(\mathrm{DHE})$, methanol electrooxidation becomes more vigorous. The loop shrinks and the Nyquist plot in Fig. 8 becomes more distorted, revealing that as well as the interface layer, the porous or rough characteristics of the electrode and the catalyst layer are produced in the methanol kinetic process by the strong methanol electrooxidation at a high applied potential. At this potential ( $0.4 \mathrm{~V}$ versus DHE), the inductance caused by $\mathrm{CO}_{\text {ads }}$ becomes less pronounced because more $\mathrm{CO}_{\mathrm{ads}}$ can react to completion than that at $0.3 \mathrm{~V}$ versus DHE. The magnitude of the arc at high frequency remains the same as that obtained at $0.3 \mathrm{~V}$ (DHE), indicating that the impedance response of this part is independent of potential and is associated with the interface. Fig. 9 presents the corresponding Bode plots and the simulation fitting at $0.4 \mathrm{~V}$ (DHE). The simulation based on the CPE-based model $\mathrm{C}$ once again matches closely the empirical results over the whole range of frequencies while that based on the model B yields large mismatches for both the arc and the loop.

As shown in Fig. 10, at $0.5 \mathrm{~V}$ (DHE), the two arcs in the Nyquist plot are severely distorted and superimposed in the interface section. The magnitude of the arc at the high-frequency end remains the same as that at $0.3 \mathrm{~V}$ (DHE), which can be seen in Fig. 5, but the arc in the low-frequency region shrinks even more. However, the third arc that is responsible for the methanol diffusion resistance is still not observed so mass-transport is not limiting at this potential. The inductance effect disappears because the reaction of the adsorbed species on the catalyst surfaces proceeds almost to completion. Although the reaction 


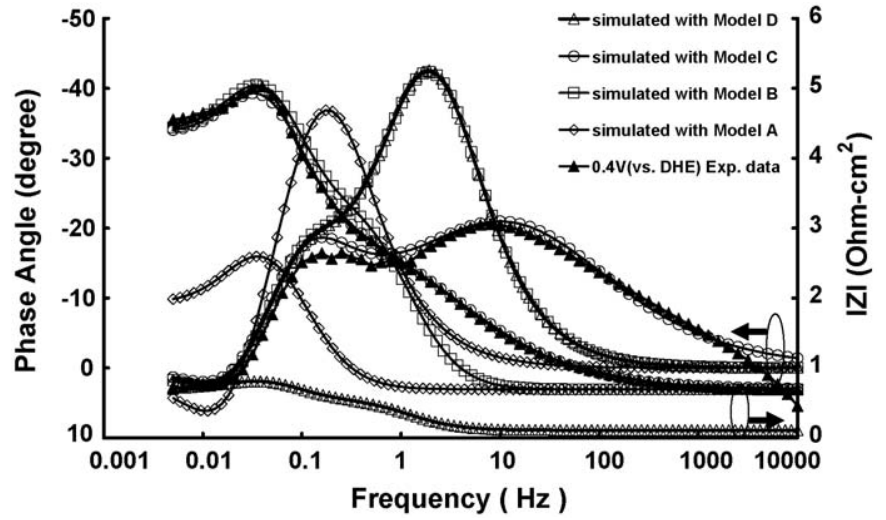

Fig. 9. Comparisons of the Bode plots of a Pt-Ru/C anode at $0.4 \mathrm{~V}$ (DHE) for experimental data and fittings of impedance models.

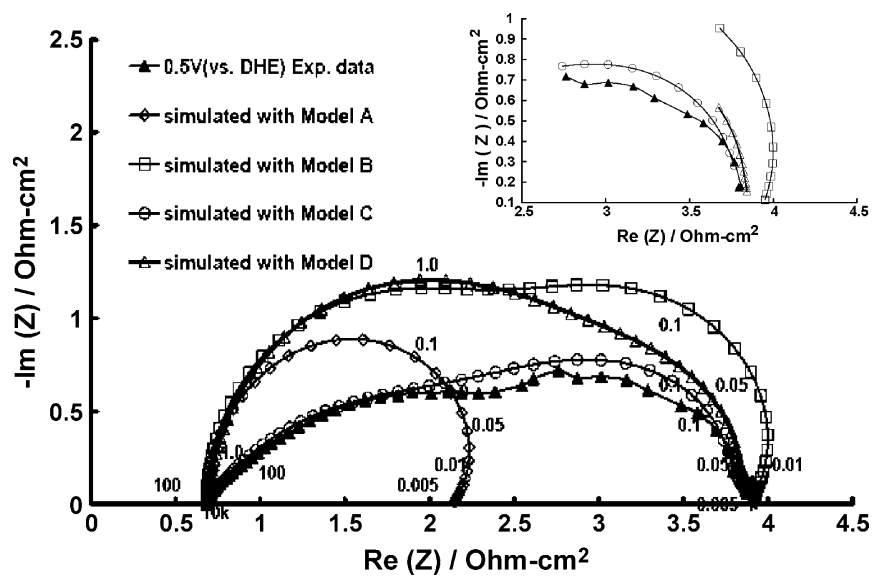

Fig. 10. Comparisons of the Nyquist impedance plots of a Pt-Ru/C anode at $0.5 \mathrm{~V}$ (DHE) for experimental data and fittings of impedance models. The inset illustrates the detailed fittings at the kinetics-controlled region.

conditions at $0.5 \mathrm{~V}$ (DHE) are more complicated than those at $0.3 \mathrm{~V}$ (DHE), the CPE-based model still provides a good fit with the experimental data, whereas the conventional model that uses capacitors rather than CPEs does not. Fig. 11 shows the corresponding Bode plots and the simulated fitting at $0.5 \mathrm{~V}$ (DHE). Furthermore, Table 2 summarizes the fitting parameters of the CPE-based equivalent-circuit at various operating potentials. In

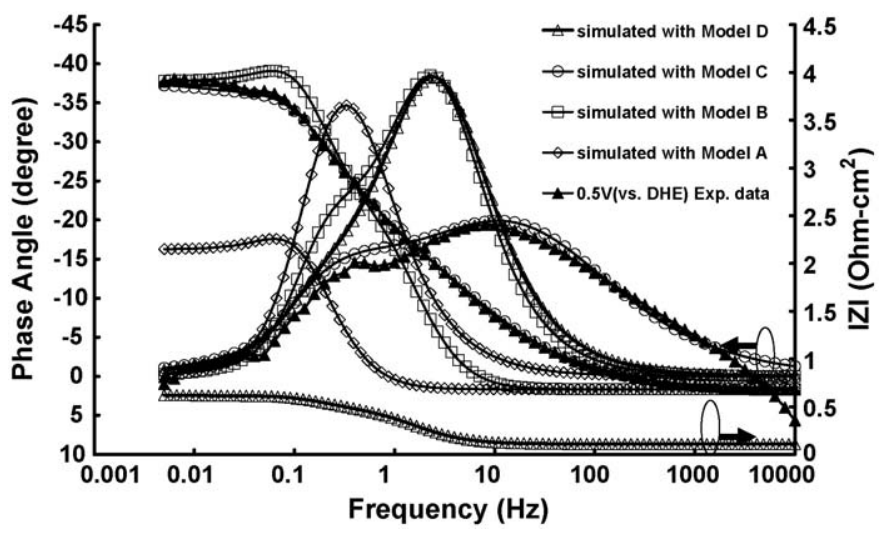

Fig. 11. Comparisons of the Bode plots a Pt-Ru/C anode at $0.5 \mathrm{~V}(\mathrm{DHE})$ for experimental data and fittings of impedance models. particular, the large deviations of the $p$ values from the ideal 1.0 indicate that the use of CPEs in the new simulation model is justified.

For all models, model $\mathrm{C}$ clearly has better fits than others in the membrane and interfaces. Although there are no apparent differences at the kinetics-controlled region, some careful analyses clearly reveal remarkable differences. It should be noted that insets of fitting details of the kinetics-controlled region are purposely added to Figs. 6, 8 and 10 to help illustrate and compare the accuracies of the models that may not be readily realized simply from the figures. By carefully compared all the simulation results, it clearly showed that model $\mathrm{C}$ is the best with respect to fitting of experimental data at the kinetics-controlled region. This clearly suggests the success of our innovative CPE-based approach to modeling a DMFC anode.

The simulations conducted by Müller et al. [6] and Liu et al. [7] of impedance in methanol electrooxidation, were based on the Faradaic processes of methanol electrooxidation at the anode, without consideration of the involvements of the anode/membrane interface and the membrane. Even given such a simplification, however, the fittings of the corresponding Nyquist plots were not close and simulated curves at high frequency did not fit the experimental results. In fact, when all three components - the anode catalyst layer, the anode/membrane interface and the membrane - were considered, the simulations that involved capacitive elements yielded results that were substantially discrepant from the experimental data, as showed by all of the Bode plots and the Nyquist plots herein. These mismatches were particularly considerable in the frequency range $1 \mathrm{~Hz}<f<100 \mathrm{~Hz}$, in which real resistance to charge transfer associated with methanol electrooxidation occurs. However, simulations using CPEs herein substantially improved fittings over the entire range of frequencies.

In fact, the phase angle versus frequency plots (Bode plots) in Figs. 6, 8 and 10 indicate the pronounced differences between the CPE- and capacitor-based simulations. When $f<0.1 \mathrm{~Hz}$, the fittings between the simulated and experimental data are good for both simulations. However, for simulations using model B, when $0.1 \mathrm{~Hz}<f<10 \mathrm{~Hz}$, the phase angle is lagged, and when $f>10 \mathrm{~Hz}$, the phase angle is overshoot. Hence, capacitors that are commonly used to represent the interface layer and the anode catalyst layer clearly depend on frequency and are not ideal circuit elements. Accordingly, these elements must be properly appropriately modified in the conventional equivalentcircuit model. Fortunately, these inconsistencies can be considerably reduced using the CPE-based equivalent-circuit model, as confirmed by the above figures. These figures indicate that when $f<0.1 \mathrm{~Hz}$, the simulation results closely fit the experimental data, for both simulations, based on CPEs or capacitors. However, when $0.1 \mathrm{~Hz}<f<1 \mathrm{~Hz},|z|$ is overestimated, but when $1 \mathrm{~Hz}<f<1000 \mathrm{~Hz},|z|$ is underestimated by simulations that use capacitors. The incorporation of CPEs in the proposed equivalent-circuit model of methanol electrochemical oxidation improves the poor fitting.

Having achieved some successes on the use of a CPE-based model, however, future studies can be carried out using delaminated membrane/electrode assemblies to emphasize the pro- 
posed contribution by the membrane interface. If porosity is the cause of the need for a CPE, experiments using different electrode porosities should confirm this assumption and possibly allow a correlation that could be used for design to be found.

\subsection{Adjustment parameter (p)}

Like the inductance effect, the values of the adjustment parameter $(p)$ provide helpful information concerning the structures of the anode/electrolyte interface and the catalyst layer. The value of $p$ in the interface layer remains constant at around 0.58 . In the main catalyst layer, $p$ varies with the applied potential. At $<0.4 \mathrm{~V}$ (DHE), $p$ equals one, indicating that the active surface reaction sites are uniformly distributed, suggesting that a relatively small fraction of catalyst participates in the electrooxidation of methanol. However, at $>0.5 \mathrm{~V}(\mathrm{DHE})$, the value of $\mathrm{p}$ decreases to around 0.76 , suggesting that the distribution of the depths of catalytic active sites is non-uniform, revealing that the current density increases with the operating potential, and the reaction rate increases, so more catalyst is required in the methanol electrooxidation. Consequently, the reaction zone expands considerably in the depth direction into the inner part of the catalyst layer and the thickness of the reactive catalyst layer increases. This fact, in turn, reflects the porosity of the catalyst particularly at high operating potentials. Therefore, both the interface and the anode unsurprisingly exhibit porous or fractal characteristics because of the discrete distribution of electrocatalyst particles.

\section{Conclusions}

The replacement of capacitive elements with CPEs supports the effective simulation of the realistic reaction conditions with porous electrode and rough interface structures. Incorporating Faradaic impedance into the interface and membrane resistances supported successful modeling of the most widely accepted reaction mechanism for methanol electrooxidation. A new equivalent circuit model, in which CPEs replace capacitive elements, was proposed. It reasonably explained the characteristic inductive behavior of a DMFC anode without limitations on mass transport. The inductance, $L_{\mathrm{CO}}$, varied markedly from around 3.9 at $0.3 \mathrm{~V}(\mathrm{DHE})$ to 0.5 at $0.5 \mathrm{~V}(\mathrm{DHE})$ as the degree of $\mathrm{CO}$ adsorption varied. The value of the adjustment parameter $\mathrm{p}$ of the interface layer remained constant at 0.58 , while that of the catalyst layer was one for $<0.4 \mathrm{~V}(\mathrm{DHE})$, decreasing to 0.76 at $0.5 \mathrm{~V}$ (DHE). The CPE-based model effectively improves the fit over the whole range of potentials of interest. Additionally, various degrees of roughness of the catalytic layer and the nonuniform distribution of the catalyst were successfully modeled and explained using CPEs.

\section{Acknowledgement}

The authors would like to thank the National Science Council of Taiwan for financially supporting this research.

\section{References}

[1] X. Zhao, X. Fan, S. Wang, S. Yang, B. Yi, Q. Xin, G. Sun, Int. J. Hydrogen Energy 30 (2005) 1003-1010.

[2] J.S. Lee, K.I. Han, S.O. Park, H.N. Kim, H. Kim, Electrochim. Acta 50 (2004) 807-810

[3] K. Furukawa, K. Okajima, M. Sudoh, J. Power Sources 139 (2005) 9-14.

[4] H. Fukunaga, T. Ishida, N. Teranishi, C. Arai, K. Yamada, Electrochim. Acta 49 (2004) 2129-3123.

[5] J.T. Mueller, P.M. Urban, J. Power Sources 75 (1998) 139-143.

[6] J.T. Müller, P.M. Urban, W.F. Holderrich, J. Power Sources 84 (1999) 157-169.

[7] Y. Liu, X. Qiu, W. Zhu, G. Wu, J. Power Sources 114 (2003) 10-14.

[8] K.S. Cole, R.H. Cole, J. Chem. Phys. 9 (1941) 341-351.

[9] V.A. Alves, L.A. da Silva, J.F.C. Boodts, Electrochim. Acta 44 (1998) 1525-1534.

[10] F. Berthier, J.P. Diard, R. Michel, J. Electroanal. Chem. 510 (2001) 1-11.

[11] S.R. Nelatury, P. Singh, J. Power Sources 112 (2002) 621-625.

[12] S.R. Nelatury, P. Singh, J. Power Sources 132 (2002) 309-314

[13] C. Liu, Q. Bi, A. Leyland, A. Matthews, Corros. Sci. 45 (2003) 1243-1256.

[14] J.M. Hu, J.Q. Zhang, C.N. Cao, Prog. Org. Coat. 46 (2003) 273-279.

[15] B. Piela, P.K. Wrona, J. Electroanal. Chem. 388 (1995) 69-79.

[16] J. Park, C. Kim, J. Bae, B. Choi, J. Jeong, B. Moon, H. Seo, I. Kim, J. Kim, Solid State Commun. 126 (2003) 635-638.

[17] S. Kochowski, K. Nitsch, B. Paszkiewicz, Thin Solid Films 444 (2003) 208-214.

[18] J.B. Wu, J. Nan, C.W. Nan, Y. Lin, Y. Deng, S. Zhao, Mater. Sci. Eng. B 99 (2003) 294-297.

[19] C. Kim, S. Pyun, J. Kim, Electrochim. Acta 48 (2003) 3455-3463.

[20] V.A. Alves, L.A. da Silva, J.F.C. Boodts, Electrochim. Acta 44 (1998) $1525-1534$

[21] X.J. Chen, K.A. Khor, S.H. Chan, J. Power Sources 123 (2003) 17-25.

[22] P.M. Faia, C.S. Furtado, A.J. Ferreira, Sens. Actuators B 107 (2005) 353-359.

[23] M. Ciureanu, H. Wang, J. Electrochem. Soc. 146 (1999) 4031-4040.

[24] J. Huslage, T. Rager, B. Schnyder, A. Tsukada, Electrochim. Acta 48 (2002) 247-254.

[25] T.E. Springer, T.A. Zawodzinski, M.S. Wilson, S. Gottesfeld, J. Electrochem. Soc. 143 (1996) 587-599.

[26] J.M. Bae, I. Honma, M. Murata, T. Yamamoto, M. Rikukawa, N. Ogata, Solid State Ionics 147 (2002) 189.

[27] J. Huslage, T. Rager, J. Kiefer, L. Steuernagel, G.G. Scherer, in: H.Z. Massoud, I. Baumvol, M. Hirose, E.H. Poindexter (Eds.), ECS Proceedings on the Micro-Power Sources, vol. 2000-3, 2001. 